Brief Report

\title{
Fully Quantum String Representation of a Wilson Loop in the Finite-Temperature 3D Yang-Mills Theory
}

\section{Dmitry Antonov}

Formerly at Departamento de Física and CFIF, Instituto Superior Técnico, ULisboa, Av. Rovisco Pais, 1049-001 Lisbon, Portugal; dr.dmitry.antonov@gmail.com

Received: 28 March 2020; Accepted: 10 April 2020; Published: 27 April 2020

\begin{abstract}
We demonstrate the emergence of the Polchinski-Strominger term in the string representation of a Wilson loop in the confinement phase of the finite-temperature 3D Yang-Mills theory. At a temperature which is roughly twice smaller than the deconfinement critical temperature, the value of the coupling of that term becomes such that the string conformal anomaly cancels out, thereby admitting a fully quantum description of the quark-antiquark string in 3D rather than 26D.
\end{abstract}

Keywords: effective string description of confinement; models of the quark-antiquark string; finite-temperature effects in quantum field theory; Wilson loop; Yang-Mills theory

\section{Introduction}

It is nowadays generally accepted that the Wilson loop of a test heavy quark in confining gauge theories should play the role of a partition function for the effective quark-antiquark string $[1,2]$. For instance, in a gauge theory with a non-Abelian symmetry group, like the Yang-Mills, the Wilson loop is defined as

$$
\langle W(C)\rangle=\frac{1}{N}\left\langle\operatorname{tr} \mathcal{P} \exp \left[i g \oint_{C} \mathrm{~d} x_{\mu} A_{\mu}(x)\right]\right\rangle .
$$

Here, "tr" is the trace over color indices, and $\mathcal{P}$ denotes the path ordering. Furthermore, $g$ is the gauge coupling, and $A_{\mu} \equiv T^{a} A_{\mu}^{a}$, where $T^{a}$ is a generator of the fundamental representation of the group $\mathrm{SU}(N) ; a=1, \ldots, N^{2}-1$, and the average $\langle\cdots\rangle$ is defined w.r.t. the action $\frac{1}{4} \int \mathrm{d}^{4} x\left(F_{\mu \nu}^{a}\right)^{2}$, where $F_{\mu \nu}^{a}=\partial_{\mu} A_{\nu}^{a}-\partial_{\nu} A_{\mu}^{a}-g f^{a b c} A_{\mu}^{b} A_{\nu}^{c}$ is the Yang-Mills field-strength tensor. For sufficiently large contours $C$, the so-called area law takes place in confining theories [3]. This law corresponds to an exponential fall-off of the Wilson loop with the area $\left|S_{\min }\right|$ of the minimal surface $S_{\min }$ bounded by the contour $C$, i.e., $\langle W(C)\rangle \rightarrow \mathrm{e}^{-\sigma\left|S_{\min }\right|}$ for $\left|S_{\min }\right| \gtrsim \frac{1}{\sigma}$. Here, the coefficient $\sigma$ of dimensionality (mass) ${ }^{2}$ is called the string tension, as it represents the energy-per-unit-length of the quark-antiquark string. The minimal surface $S_{\min }$ is unambiguously defined by the contour $C$, regardless of whether that contour is flat or not. Being the world sheet of the maximally stretched confining string, the minimal surface should play a distinguished role in the representation of the Wilson loop in the form of an integral over all surfaces $S$ bounded by the contour $C$. For the case of $\left|S_{\min }\right| \gtrsim \frac{1}{\sigma}$ at issue, such an integral representation reads

$$
\langle W(C)\rangle \simeq \mathrm{e}^{-\sigma\left|S_{\min }\right|}=\int \mathrm{d} \mu(S) \mathrm{e}^{-\mathcal{A}(S)}
$$

Here, $\mu(S)$ and $\mathcal{A}(S)$ are, respectively, some integration measure and action associated with the surface $S$. In the physically mostly interesting case of the Yang-Mills theory, by using the non-Abelian 
Stokes' theorem and the cumulant expansion truncated at the second term, one can approximate Wilson loop (1) as [4-6]

$$
\langle W(C)\rangle \simeq \frac{1}{N} \operatorname{tr} \exp \left[-\frac{1}{2^{2} 2 !} \int_{S_{\min }} \mathrm{d} \sigma_{\mu_{1} v_{1}}\left(x_{1}\right) \int_{S_{\min }} \mathrm{d} \sigma_{\mu_{2} v_{2}}\left(x_{2}\right)\left\langle g^{2} F_{\mu_{1} v_{1}}\left(x_{1}\right) F_{\mu_{2} v_{2}}\left(x_{2}\right)\right\rangle\right] .
$$

The integration on the r.h.s. of this expression should be performed over $S_{\min }$, since otherwise an artificial dependence on an arbitrary surface $S$ appears; only by retaining all the terms of the cumulant expansion may one keep $S$ instead of $S_{\min }$ on the r.h.s. Furthermore, on $S_{\min }$, higher-derivative terms in the derivative expansion of this expression vanish, and only the Nambu-Goto term remains, yielding $\mathrm{e}^{-\sigma\left|S_{\min }\right|}$. Accordingly, measure $\mathrm{d} \mu(S)$ in this case has the form $\mathrm{d} \mu(S)=\delta\left(S-S_{\min }\right) \mathrm{d} S$. While retaining in the action $\mathcal{A}(S)$ only the quadratic cumulant, the expression for the measure becomes less trivial once we account for the effect of the next, quartic, cumulant which contributes to the action of the quark-antiquark string:

$$
\begin{gathered}
\langle W(C)\rangle \simeq \frac{1}{N} \operatorname{tr} \exp \left[-\frac{1}{2^{2} 2 !} \int_{S_{\min }} \mathrm{d} \sigma_{\mu_{1} v_{1}}\left(x_{1}\right) \int_{S_{\text {min }}} \mathrm{d} \sigma_{\mu_{2} v_{2}}\left(x_{2}\right)\left\langle g^{2} F_{\mu_{1} v_{1}}\left(x_{1}\right) F_{\mu_{2} v_{2}}\left(x_{2}\right)\right\rangle\right. \\
+\frac{1}{2^{4} 4 !} \int_{S_{\min }} \mathrm{d} \sigma_{\mu_{1} v_{1}}\left(x_{1}\right) \int_{S_{\min }} \mathrm{d} \sigma_{\mu_{2} v_{2}}\left(x_{2}\right) \int_{S_{\min }} \mathrm{d} \sigma_{\mu_{3} v_{3}}\left(x_{3}\right) \int_{S_{\min }} \mathrm{d} \sigma_{\mu_{4} v_{4}}\left(x_{4}\right) \times \\
\left.\times\left\langle g^{4} F_{\mu_{1} v_{1}}\left(x_{1}\right) F_{\mu_{2} v_{2}}\left(x_{2}\right) F_{\mu_{3} v_{3}}\left(x_{3}\right) F_{\mu_{4} v_{4}}\left(x_{4}\right)\right\rangle_{c}\right]
\end{gathered}
$$

where the subscript " $c$ " stands for "connected" to distinguish the cumulant from the standard 4-point correlation function. The corresponding expression for the measure then reads

$$
\begin{aligned}
\mathrm{d} \mu(S)=\delta\left(S-S_{\min }\right) & \exp \left[-\frac{1}{2^{4} 4 !} \int_{S} \mathrm{~d} \sigma_{\mu_{1} v_{1}}\left(x_{1}\right) \int_{S} \mathrm{~d} \sigma_{\mu_{2} v_{2}}\left(x_{2}\right) \int_{S} \mathrm{~d} \sigma_{\mu_{3} v_{3}}\left(x_{3}\right) \int_{S} \mathrm{~d} \sigma_{\mu_{4} v_{4}}\left(x_{4}\right) \times\right. \\
& \left.\times\left\langle g^{4} F_{\mu_{1} v_{1}}\left(x_{1}\right) F_{\mu_{2} v_{2}}\left(x_{2}\right) F_{\mu_{3} v_{3}}\left(x_{3}\right) F_{\mu_{4} v_{4}}\left(x_{4}\right)\right\rangle_{c}\right] \mathrm{d} S .
\end{aligned}
$$

In this paper, we show that accounting for the quartic cumulant in the finite-temperature 3D Yang-Mills theory yields the so-called Polchinski-Strominger term [7] in the string representation of a Wilson loop, which allows one to cancel string conformal anomaly at a particular temperature. As is well known (see e.g., [8]), the conformal anomaly emerges in the course of quantization of the Nambu-Goto string, thereby making quantization of the bosonic string in the space-time of any dimensionality other than 26 impossible.

Formally, our observation is as follows. The Nambu-Goto string action has the form $\mathcal{A}_{\mathrm{NG}}(S)=$ $\sigma \int \mathrm{d}^{2} \xi \sqrt{\operatorname{det} g_{a b}}$, where $g_{a b}=\partial_{a} x_{\mu} \cdot \partial_{b} x_{\mu}$ is the tensor of the induced metric corresponding to the vector-function $x_{\mu}=x_{\mu}(\xi)$, where the latter parameterizes the string world sheet $S$. Here, indices $a$ and $b$ take the values 1 and 2 , and $\xi=\left(\xi_{1}, \xi_{2}\right)$ is a 2 D-vector of flat dimensionless coordinates, while index $\mu$ takes the values $1, \ldots, D$, where $D$ is the dimensionality of the embedding Euclidean space-time. The Nambu-Goto string action is semiclassically equivalent to the Polyakov string action [8] $\mathcal{A}_{\mathrm{P}}=\frac{\sigma}{2} \int \mathrm{d}^{2} \xi \sqrt{\gamma} \gamma^{a b} g_{a b}$, where $\gamma_{a b}$ is an auxiliary metric, and $\gamma \equiv \operatorname{det} \gamma_{a b}$. (To prove this equivalence, one can use the following expression for the variation of the determinant of the metric: $\delta \gamma=-\gamma \cdot \gamma_{a b} \cdot \delta \gamma^{a b}$, which yields $\delta \mathcal{A}_{\mathrm{P}}=\frac{\sigma}{2} \int \mathrm{d}^{2} \xi \sqrt{\gamma} \cdot \delta \gamma^{a b} \cdot T_{a b}$, where $T_{a b}=g_{a b}-\frac{1}{2} g_{c d} \gamma^{c d} \gamma_{a b}$ is the energy-momentum tensor. The stationary point in the functional integral over auxiliary metrics is defined by the corresponding classical equation of motion, $T_{a b}=0$. For the solution of this equation, which is $\gamma_{a b}=g_{a b}$, the Polyakov action $\mathcal{A}_{\mathrm{P}}$ is indeed equal to the Nambu-Goto action $\mathcal{A}_{\mathrm{NG}}$.) Due to this equivalence, the string quantization can be performed by integrating over $x_{\mu}$, which returns the string partition function in the form of a functional integral over $\gamma_{a b}$. In the course of the quantization, one notices that, since the Polyakov action stays invariant under the reparametrizations of the surface, one has to fix some gauge in the group of reparametrizations. Such a gauge fixing results in the 
additional integration over the ghost fields. Usually, one adopts the so-called conformal gauge, in which the metric $\gamma_{a b}$ has the diagonal form, namely $\gamma_{a b}=\sqrt{\gamma} \delta_{a b}$. In this gauge, the Polyakov action takes the form $\mathcal{A}_{\mathrm{P}}=\frac{\sigma}{2} \int \mathrm{d}^{2} \xi\left(\partial_{a} x_{\mu}\right)^{2}$, which is just the theory of a free massless bosonic field $x_{\mu}$. Integrating then over $x_{\mu}$, one arrives at a Liouville theory,

$$
\int \mathcal{D} x_{\mu} \mathrm{e}^{-\mathcal{A}_{\mathrm{P}}}=\mathrm{e}^{\frac{D}{48 \pi} \int \mathrm{d}^{2} \xi\left[\frac{1}{2}\left(\partial_{a} \varphi\right)^{2}+\mu^{2} \mathrm{e}^{\varphi}\right]}
$$

where $\varphi=\frac{1}{2} \ln \gamma$ and $\mu^{2}=\frac{48 \pi \sigma}{26-D}$. Integrating further over the ghosts, one obtains for the corresponding Faddeev-Popov determinant a parametrically similar result: $\mathrm{e}^{-\frac{26}{48 \pi} \int \mathrm{d}^{2} \xi\left[\frac{1}{2}\left(\partial_{a} \varphi\right)^{2}+\mu^{2} \mathrm{e}^{\varphi}\right]}$. Bringing these two expressions together, one arrives at the following expression for the string partition function [8]:

$$
\mathcal{Z}=\int \mathcal{D} \varphi \mathrm{e}^{-\frac{26-D}{48 \pi} \int \mathrm{d}^{2} \xi\left[\frac{1}{2}\left(\partial_{a} \varphi\right)^{2}+\mu^{2} \mathrm{e}^{\varphi}\right]} .
$$

This result means that the conformal anomaly cancels out and the bosonic string can be selfconsistently quantized only for $D=26$. In this respect, the Nambu-Goto model of the bosonic string drastically differs from the field-theoretical models of point particles, which can be quantized in the space-time of any dimensionality as long as their renormalizability is provided. For this reason, being used as a model of the hadronic string, the Nambu-Goto string admits at best a semiclassical treatment. This yields the so-called Lüscher term [9], or even the full static potential in the large- $D$ limit [10], but the fully quantum treatment of the string remains missing.

An approach enabling the Nambu-Goto string to remain quantizable in the space-time of dimensionality $D$ other than 26 is based on the observation that the partition function (4) can be represented in the form

$$
\mathcal{Z}=\int \mathcal{D} \varphi \mathrm{e}^{-\sigma \int \mathrm{d}^{2} \xi \sqrt{\gamma}-\frac{26-D}{96 \pi} \int \mathrm{d}^{2} \xi \int \mathrm{d}^{2} \xi^{\prime} \sqrt{\gamma} R\left(-\frac{1}{\partial^{2}}\right)_{\xi, \xi^{\prime}} \sqrt{\gamma^{\prime}} R^{\prime}}
$$

Here, $\partial^{2} \equiv \partial^{a} \partial_{a}$ and $R=-\mathrm{e}^{-\varphi} \partial^{2} \varphi$ are the conformal-gauge expressions for the Laplacian $\Delta=\frac{1}{\sqrt{\gamma}} \partial_{a} \sqrt{\gamma} \gamma^{a b} \partial_{b}$ and the scalar curvature of the world sheet, respectively; $\left(-\frac{1}{\partial^{2}}\right)_{\xi, \xi^{\prime}}=\frac{1}{2 \pi} \ln \frac{1}{\left|\xi-\xi^{\prime}\right|}$ is the Green's function of the Laplacian; $\gamma^{\prime}=\gamma\left(\boldsymbol{\xi}^{\prime}\right), R^{\prime}=R\left(\boldsymbol{\xi}^{\prime}\right)$. Given the semiclassical equality $\gamma_{a b}=g_{a b}$ (cf. the discussion above), one can say that, starting with an extension of the Nambu-Goto model by a non-local term $\kappa \int \mathrm{d}^{2} \xi \int \mathrm{d}^{2} \xi^{\prime} \sqrt{\gamma} R\left(-\frac{1}{\partial^{2}}\right)_{\xi, \xi^{\prime}} \sqrt{\gamma^{\prime}} R^{\prime}$, one can hope to have the situation with

$$
\kappa=\frac{D-26}{96 \pi} .
$$

Such a value of $\kappa$ would make the corresponding string model quantizable in the space-time of dimensionality $D$. The desired term can be viewed as a non-trivial $2 \mathrm{D}$ theory of gravity, while Einstein-Hilbert gravity is trivial in 2D, since $\int d^{2} \xi \sqrt{\gamma} R$ is a full derivative. Being represented in the form $-\kappa \int \mathrm{d}^{2} \xi\left(\partial_{a} \ln \sqrt{\gamma}\right)^{2}$, the desired non-local term is usually referred to as the Polchinski-Strominger (PS) term [7]. This term could appear by means of the integration over some scalar field $\Phi(\xi)$ coupled to the string world sheet as $\int \mathrm{d}^{2} \xi \Phi \sqrt{g} R$, where from now on $g \equiv \operatorname{det} g_{a b}$ and $R=R\left[g_{a b}\right]$. However, in the case of the Yang-Mills theory of interest, it is not clear where such a scalar field existing only on the string world sheet could stem from.

Here, we put forward an alternative observation that the PS term in the string representation of the Wilson loop can emerge due to the quartic cumulant, since this term can be viewed as a non-local square of the Euler characteristic of the world sheet, $\chi=\frac{1}{4 \pi} \int \mathrm{d}^{2} \xi \sqrt{g} R$. To come closer to this observation, let us recall the emergence of the Euler characteristic in the course of the derivative 
expansion which involves only the quadratic cumulant. To that end, we parameterize the part of the quadratic cumulant which yields the surface $\times$ surface interaction, as [4-6]

$$
\left\langle g^{2} F_{\mu_{1} v_{1}}^{a}\left(x_{1}\right) F_{\mu_{2} v_{2}}^{b}\left(x_{2}\right)\right\rangle=\left\langle\left(g F_{\mu \nu}^{a}\right)^{2}\right\rangle \frac{\delta^{a b}}{\left(N^{2}-1\right)\left(D^{2}-D\right)}\left(\delta_{\mu_{1} \mu_{2}} \delta_{v_{1} \nu_{2}}-\delta_{\mu_{1} v_{2}} \delta_{\mu_{2} v_{1}}\right) \mathcal{D}\left(x_{1}-x_{2}\right) .
$$

Here, the dimensionless function $\mathcal{D}(x)$ obeys the normalization condition $\mathcal{D}(0)=1$ and falls off at least exponentially fast at a distance equal to the correlation length $\lambda \equiv 1 / m$ of the Yang-Mills vacuum, e.g., $\mathcal{D}(x)=\mathrm{e}^{-m|x|}$. The corresponding contribution to the Wilson loop reads

$$
\begin{gathered}
\langle W(C)\rangle \simeq \frac{1}{N} \operatorname{tr} \exp \left[-\frac{1}{2^{2} 2 !} \int_{S_{\min }} \mathrm{d} \sigma_{\mu_{1} v_{1}}\left(x_{1}\right) \int_{S_{\min }} \mathrm{d} \sigma_{\mu_{2} v_{2}}\left(x_{2}\right)\left\langle g^{2} F_{\mu_{1} v_{1}}^{a}\left(x_{1}\right) F_{\mu_{2} v_{2}}^{b}\left(x_{2}\right)\right\rangle T^{a} T^{b}\right]= \\
=\exp \left[-\frac{\left\langle\left(g F_{\mu \nu}^{a}\right)^{2}\right\rangle}{8 N\left(D^{2}-D\right)} \int_{S_{\min }} \mathrm{d} \sigma_{\mu v}\left(x_{1}\right) \int_{S_{\min }} \mathrm{d} \sigma_{\mu v}\left(x_{2}\right) \mathcal{D}\left(x_{1}-x_{2}\right)\right] .
\end{gathered}
$$

One can further perform the derivative expansion $[11,12]$ of the corresponding action

$$
\mathcal{A}(S)=\int_{S} \mathrm{~d} \sigma_{\mu v}\left(x_{1}\right) \int_{S} \mathrm{~d} \sigma_{\mu v}\left(x_{2}\right) \tilde{\mathcal{D}}\left(x_{1}-x_{2}\right), \text { where } \tilde{\mathcal{D}}(x) \equiv \frac{\left\langle\left(g F_{\mu v}^{a}\right)^{2}\right\rangle}{8 N\left(D^{2}-D\right)} \mathcal{D}(x) .
$$

A useful relation, which is utilized to perform the expansion, is the so-called Gauss-Weingarten formula $D_{a} D_{b} x_{\mu}=K_{a b}^{i} n_{\mu}^{i}$ for the covariant derivative $D_{a} D_{b} x_{\mu} \equiv D_{a} \partial_{b} x_{\mu}=\partial_{a} \partial_{b} x_{\mu}-\Gamma_{a b}^{c} \partial_{c} x_{\mu}$. This formula enables one to replace the products of ordinary derivatives $\partial_{a} \partial_{b} x_{\mu}$ by the products of covariant derivatives $D_{a} D_{b} x_{\mu}$. Here, $\Gamma_{a b}^{c}$ is a Christoffel symbol defined w.r.t. the induced metric $g_{a b}, n_{\mu}^{i}$ 's are the unit normal vectors to the world sheet, $K_{a b}^{i}$ is the second fundamental form of the world sheet, and index $i$ takes the values $1, \ldots, D-2$. The normal vectors $n_{\mu}^{i}$ 's obey the condition $n_{\mu}^{i} \cdot \partial_{a} x_{\mu}=0$, which yields the following orthogonality relation: $D_{a} D_{b} x_{\mu} \cdot \partial_{c} x_{\mu}=0$. In particular, with the use of this relation, one can prove a complete mutual cancellation of the terms proportional to $\int \mathrm{d}^{2} \xi\left(\partial_{a} \ln \sqrt{g}\right)^{2}$, to the unique order $\mathcal{O}\left(\lambda^{4}\right)$ in which these terms could appear. This observation obviously means that the derivative expansion does not yield the PS term and, consequently, it does not provide the possibility to cancel the conformal anomaly for any value of $D$ other than 26 . By using the above formulae along with the orthonormality relation $n_{\mu}^{i} n_{\mu}^{j}=\delta^{i j}$, it is also possible to convert the products of expressions $D_{a} D_{b} x_{\mu}$, emerging in the course of the derivative expansion, into the products of the second fundamental form, e.g., as follows:

$$
\left(g^{a b} g^{c d}+g^{a d} g^{b c}+g^{a c} g^{b d}\right) D_{a} D_{b} x_{\mu} \cdot D_{c} D_{d} x_{\mu}=3\left(\partial^{2} x_{\mu}\right)^{2}+2\left(K_{a b}^{i} K^{i, a b}-K_{a}^{i a} K_{b}^{i b}\right) .
$$

One can further use the relation $K_{a b}^{i} K^{i, a b}-K_{a}^{i a} K_{b}^{i b}=-R$ to obtain all the terms which yield the Euler characteristic $\chi$. Altogether, to the order $\mathcal{O}\left(\lambda^{4}\right)$, the derivative expansion yields:

$$
\mathcal{A}(S) \simeq \sigma \int \mathrm{d}^{2} \xi \sqrt{g}+\frac{1}{\alpha} \int \mathrm{d}^{2} \xi \sqrt{g}\left(\partial^{2} x_{\mu}\right)^{2}+\beta \int \mathrm{d}^{2} \xi \sqrt{g} R,
$$

where

$$
\sigma=2 \lambda^{2} \int \mathrm{d}^{2} z \tilde{\mathcal{D}}(\mathbf{z}), \frac{1}{\alpha}=-\frac{\lambda^{4}}{8} \int \mathrm{d}^{2} z \mathbf{z}^{2} \tilde{\mathcal{D}}(\mathbf{z}), \text { and } \beta=\frac{\lambda^{4}}{12} \int \mathrm{d}^{2} z \mathbf{z}^{2} \tilde{\mathcal{D}}(\mathbf{z})
$$

are the string tension, the coupling of the rigidity term, and the coefficient at $4 \pi \chi$, respectively. The leading terms that have been omitted in Equation (6) are those whose coefficients are proportional to the next even-order integral moment of the function $\tilde{\mathcal{D}}$, i.e., these coefficients have the order of $\lambda^{8} \int \mathrm{d}^{2} z|\mathbf{z}|^{4} \tilde{\mathcal{D}}(\mathbf{z})$. Altogether, the derivative expansion converges provided that $\lambda \lesssim \sqrt{\left|S_{\min }\right|}$, which means that confinement in the Yang-Mills theory takes place and admits an effective string description as long as the quark-antiquark separation stays larger than the vacuum correlation length $\lambda$. 
In the next section, we will see how the PS term in the derivative expansion can emerge from the quartic cumulant in Equation (3) in the case of the finite-temperature 3D Yang-Mills theory.

\section{PS Term from the Quartic Cumulant}

We consider only the part of the quartic cumulant, which yields in Equation (3) four surface integrals, none of which can be reduced to the contour integral by means of the (Abelian) Stokes' theorem. That part does not contain derivatives in its tensor structure, and can be parameterized as follows [13]:

$$
\begin{gathered}
\left\langle g^{4} F_{\mu_{1} v_{1}}^{a_{1}}\left(x_{1}\right) F_{\mu_{2} v_{2}}^{a_{2}}\left(x_{2}\right) F_{\mu_{3} v_{3}}^{a_{3}}\left(x_{3}\right) F_{\mu_{4} v_{4}}^{a_{4}}\left(x_{4}\right)\right\rangle_{c}=C\left\langle\left(g^{2} F_{\mu v}^{a} F_{\mu v}^{a}\right)^{2}\right\rangle \times \\
\times\left[\delta^{a_{1} a_{2}} \delta^{a_{3} a_{4}}\left(\delta_{\mu_{1} \mu_{2}} \delta_{v_{1} v_{2}}-\delta_{\mu_{1} v_{2}} \delta_{\mu_{2} v_{1}}\right)\left(\delta_{\mu_{3} \mu_{4}} \delta_{v_{3} v_{4}}-\delta_{\mu_{3} v_{4}} \delta_{\mu_{4} v_{3}}\right)+\right. \\
+\delta^{a_{1} a_{3}} \delta^{a_{2} a_{4}}\left(\delta_{\mu_{1} \mu_{3}} \delta_{v_{1} v_{3}}-\delta_{\mu_{1} v_{3}} \delta_{\mu_{3} v_{1}}\right)\left(\delta_{\mu_{2} \mu_{4}} \delta_{v_{2} v_{4}}-\delta_{\mu_{2} v_{4}} \delta_{\mu_{4} v_{2}}\right)+ \\
\left.+\delta^{a_{1} a_{4}} \delta^{a_{2} a_{3}}\left(\delta_{\mu_{1} \mu_{4}} \delta_{v_{1} v_{4}}-\delta_{\mu_{1} v_{4}} \delta_{\mu_{4} v_{1}}\right)\left(\delta_{\mu_{2} \mu_{3}} \delta_{v_{2} v_{3}}-\delta_{\mu_{2} v_{3}} \delta_{\mu_{3} v_{2}}\right)\right] \mathrm{e}^{-m\left(\left|z_{12}\right|+\left|z_{13}\right|+\left|z_{14}\right|+\left|z_{23}\right|+\left|z_{24}\right|+\left|z_{34}\right|\right) .}
\end{gathered}
$$

Here, $z_{i j}=x_{i}-x_{j}$ are the relative coordinates of the points $x_{1}, \ldots, x_{4}$, so that the whole expression vanishes with the increase of any of the corresponding relative distances $\left|z_{i j}\right|$, as it should be for a cumulant. Under the assumption that the amplitude of the listed tensor structure significantly exceeds the amplitudes of all the omitted structures, which contain derivatives and therefore do not contribute to the string tension and, hence, to confinement (This assumption is motivated by the corresponding lattice results $[14,15]$ for the quadratic cumulant.), one can further readily find the normalization coefficient: $C=\left\{\left(N^{2}-1\right)\left(D^{2}-D\right)\left[\left(N^{2}-1\right)\left(D^{2}-D\right)+4\right]\right\}^{-1}$. Accordingly, the correction produced to the string action by the quartic cumulant (8) reads

$$
\begin{gathered}
\mathcal{A}(S)-\int_{S} \mathrm{~d} \sigma_{\mu v}\left(x_{1}\right) \int_{S} \mathrm{~d} \sigma_{\mu v}\left(x_{2}\right) \tilde{\mathcal{D}}\left(x_{1}-x_{2}\right)= \\
=-\frac{C}{2^{4} 4 !}\left\langle\left(g^{2} F_{\mu v}^{a} F_{\mu v}^{a}\right)^{2}\right\rangle \frac{1}{N} \operatorname{tr} \prod_{k=1}^{4} \int_{S} \mathrm{~d} \sigma_{\mu_{k} v_{k}}\left(x_{k}\right) T^{a_{k}}[\cdots] \mathrm{e}^{-m(\cdots)}=-\frac{C}{2^{4} 4 !}\left\langle\left(g^{2} F_{\mu \nu}^{a} F_{\mu v}^{a}\right)^{2}\right\rangle\left(\frac{N^{2}-1}{2 N}\right)^{2} \times \\
\times 4 \int_{S} \int_{S} \int_{S} \int_{S}\left[\mathrm{~d} \sigma_{\mu v}\left(x_{1}\right) \mathrm{d} \sigma_{\mu v}\left(x_{2}\right) \mathrm{d} \sigma_{\lambda \rho}\left(x_{3}\right) \mathrm{d} \sigma_{\lambda \rho}\left(x_{4}\right)+\mathrm{d} \sigma_{\mu v}\left(x_{1}\right) \mathrm{d} \sigma_{\mu v}\left(x_{3}\right) \mathrm{d} \sigma_{\lambda \rho}\left(x_{2}\right) \mathrm{d} \sigma_{\lambda \rho}\left(x_{4}\right)+\right. \\
\left.+\mathrm{d} \sigma_{\mu \nu}\left(x_{1}\right) \mathrm{d} \sigma_{\mu v}\left(x_{4}\right) \mathrm{d} \sigma_{\lambda \rho}\left(x_{2}\right) \mathrm{d} \sigma_{\lambda \rho}\left(x_{3}\right)\right] \mathrm{e}^{-m\left(\left|z_{12}\right|+\left|z_{13}\right|+\left|z_{14}\right|+\left|z_{23}\right|+\left|z_{24}\right|+\left|z_{34}\right|\right) .}
\end{gathered}
$$

We proceed now to the 3D Yang-Mills, where the coupling $g$ has dimensionality (mass) $)^{1 / 2}$. There, being inspired by the exact result for the function $\mathcal{D}(\vec{x})$ in the London limit of the dual superconductor or in the 3D compact QED, which is proportional to the propagator of a dual gauge boson [11,16], we replace $\mathrm{e}^{-m\left|z_{i j}\right|}$ in Equation (8) by $\mathrm{e}^{-m\left|\vec{z}_{i j}\right|} /\left(m\left|\vec{z}_{i j}\right|\right)$. We notice that the relative coefficient between these two functions should stay equal to 1 , since $\int \mathrm{d}^{2} x \mathrm{e}^{-m|\mathrm{x}|}=\int \mathrm{d}^{2} x \frac{\mathrm{e}^{-m|x|}}{m|\mathrm{x}|}$, which means that the corresponding replacement in the function $\mathcal{D}(\vec{x})$ would leave the string tension unchanged. Moreover, we consider the 3D Yang-Mills at finite temperature T, which leads to the decomposition $\frac{\mathrm{e}^{-m|\vec{x}|}}{|\vec{x}|}=$ $2 T \sum_{n=-\infty}^{+\infty} K_{0}\left(m|\mathbf{x}| \sqrt{1+\left(\omega_{n} / m\right)^{2}}\right)$, where $\omega_{n}=2 \pi T n$ is the $n^{\prime}$ s Matsubara frequency, and $K_{0}(x)$ is the Macdonald function. Due to the exponential fall-off of $K_{0}(x)$ at large $x$, it suffices to keep in the above sum only the zeroth term, which yields $\frac{\mathrm{e}^{-m|\vec{x}|}}{|\vec{x}|} \simeq 2 T K_{0}(m|\mathbf{x}|)$ at temperatures $T \gtrsim \frac{m}{2 \pi}$. We have then e.g., for the first term on the r.h.s. of Equation (9) the following expression:

$$
4 \int_{S} \int_{S} \int_{S} \int_{S} \mathrm{~d} \sigma_{\mu v}\left(\vec{x}_{1}\right) \mathrm{d} \sigma_{\mu v}\left(\vec{x}_{2}\right) \mathrm{d} \sigma_{\lambda \rho}\left(\vec{x}_{3}\right) \mathrm{d} \sigma_{\lambda \rho}\left(\vec{x}_{4}\right)\left(\frac{2 T}{m}\right)^{6} K_{0}\left(m\left|\mathbf{z}_{12}\right|\right) \cdot K_{0}\left(m\left|\mathbf{z}_{34}\right|\right) \cdot \frac{1}{4} K_{0}\left(4 m\left|\mathbf{z}_{13}\right|\right),
$$

where we have used the proximity of $\vec{x}_{1}$ to $\vec{x}_{2}$ and of $\vec{x}_{3}$ to $\vec{x}_{4}$ to approximate $\mathrm{e}^{-m\left(\left|\vec{z}_{13}\right|+\left|\vec{z}_{14}\right|+\left|\vec{z}_{23}\right|+\left|\vec{z}_{24}\right|\right)}$ by $\mathrm{e}^{-4 m\left|\vec{z}_{13}\right|}$ and, consequently, by $\frac{2 T}{4 m} K_{0}\left(4 m\left|\mathbf{z}_{13}\right|\right)$. By using further the value of coefficient $\beta$ from 
Equation (7), we find that the above expression yields, in particular, the following contribution to the string action:

$$
\left(\frac{4 \pi}{3}\right)^{2} \lambda^{8}\left(\frac{2 T}{m}\right)^{6} \frac{1}{4} \int \mathrm{d}^{2} \xi \int \mathrm{d}^{2} \xi^{\prime} \sqrt{g(\mathbf{x})} R(\mathbf{x}) K_{0}\left(4 m\left|\mathbf{x}-\mathbf{x}^{\prime}\right|\right) \sqrt{g\left(\mathbf{x}^{\prime}\right)} R\left(\mathbf{x}^{\prime}\right),
$$

where $\frac{4 \pi}{3}=\frac{1}{6} \int \mathrm{d}^{2} z \mathbf{z}^{2} K_{0}(|\mathbf{z}|), \mathbf{x} \equiv \mathbf{x}(\boldsymbol{\xi}), \mathbf{x}^{\prime} \equiv \mathbf{x}\left(\boldsymbol{\xi}^{\prime}\right)$. Furthermore, it is natural to parameterize $\mathbf{x}$ as $\mathbf{x}=L \xi$, where the parameter $L$ has the dimensionality of length. (For example, for a flat circular contour $C$, the parameter $L$ is just the radius of $C$, while $\xi$ is the vector parameterizing the disc of radius 1.) As is known [8], string conformal anomaly occurs at short distances, $\xi \rightarrow \xi^{\prime}$, where $K_{0}\left(4 m\left|\mathbf{x}-\mathbf{x}^{\prime}\right|\right) \rightarrow \ln \frac{1}{4 m L\left|\xi-\xi^{\prime}\right|} \rightarrow 2 \pi\left(-\frac{1}{\partial^{2}}\right)_{\xi, \xi^{\prime \prime}}$ which yields the PS term.

To calculate the PS-term coupling $\kappa$ in terms of $g^{2}$ and $T$, we use the following expressions, which are exact in the 3D Yang-Mills [17,18]: $m=\frac{g^{2} N}{2 \pi}$ and $\sigma=g^{4} \frac{N^{2}-1}{8 \pi}$. On the other hand, by using Equation (7) with $\mathcal{D}(\mathbf{z})=\frac{\mathrm{e}^{-|\mathbf{z}|}}{|\mathbf{z}|}$, we have $\sigma=\frac{\pi}{12 N} \frac{\left\langle\left(g F_{\mu \nu}^{a}\right)^{2}\right\rangle}{m^{2}}$, which yields $\left\langle\left(g F_{\mu \nu}^{a}\right)^{2}\right\rangle=\frac{3}{2 \pi^{2}} N\left(N^{2}-\right.$ 1) $m^{2} g^{4}$. Having noticed that both sides of this expression consistently scale at large $N$ as $\mathcal{O}(N)$, we henceforth set $N=3$. Furthermore, to evaluate $\left\langle\left(g^{2} F_{\mu \nu}^{a} F_{\mu \nu}^{a}\right)^{2}\right\rangle$, we use the so-called factorization hypothesis, also called vacuum dominance [19], which states that the dominant contribution to condensates like $\left\langle\left(g^{2} F_{\mu \nu}^{a} F_{\mu \nu}^{a}\right)^{2}\right\rangle$ is the factorized one: $\left\langle\left(g^{2} F_{\mu \nu}^{a} F_{\mu \nu}^{a}\right)^{2}\right\rangle \simeq\left\langle\left(g F_{\mu \nu}^{a}\right)^{2}\right\rangle^{2}$. Bringing everything together, we have: $\kappa=-\frac{16}{39 \pi} \frac{g^{8} T^{6}}{m^{10}}$. Equating this expression to Equation (5) at $D=3$, and substituting $m=\frac{3 g^{2}}{2 \pi}$, we obtain the value of the temperature at which the string conformal anomaly cancels out:

$$
T_{*}=\left(\frac{299}{512}\right)^{1 / 6}\left(\frac{3}{2 \pi}\right)^{5 / 3} \cdot g^{2} \simeq 0.267 g^{2} .
$$

Comparing this expression with $\frac{m}{2 \pi} \simeq 0.076 g^{2}$, we notice that $T_{*}$ exceeds $\frac{m}{2 \pi}$ by a factor $\simeq 3.5$, thereby justifying the above-used approximation of the sum over Matsubara modes by its zeroth term. On the other hand, one can compare $T_{*}$ with the available lattice data for the deconfinement critical temperature $T_{c}$ in the 3D Yang-Mills. According to Refs. [20,21], $T_{\mathcal{c}} \simeq \sqrt{\sigma}=\frac{g^{2}}{\sqrt{\pi}} \simeq 0.56 g^{2}$, which is larger than $T_{*}$ by a factor $\simeq 2.1$. This check confirms that the obtained possible cancellation of string conformal anomaly occurs "deeply enough" in the confinement phase, where the effects of string broadening, which take place at $T \rightarrow T_{\mathcal{C}}$, can be safely disregarded.

\section{Summary}

In conclusion, we have shown that the PS term can emerge in the string representation of the Wilson loop in the confinement phase of the finite-temperature 3D Yang-Mills theory, with the origin of that term being the quartic cumulant (8). At the particular temperature (10), the value of the coupling of the PS term becomes such that this term can lead to the cancellation of the bosonic-string conformal anomaly, thereby providing a fully quantum description of the quark-antiquark string at that temperature (as opposed to the well-known semiclassical treatments of the Nambu-Goto quark-antiquark string $[9,10]$, which account for small fluctuations of the string around its classical configuration). It is, however, worth noticing that the suggested approach is specific for the 3D case at finite temperature, where the surface $\times$ surface interaction in the zeroth-Matsubara-mode approximation becomes two-dimensional. For this reason, it is unfortunately not applicable to the physically mostly interesting case of the 4D Yang-Mills at zero temperature, where other possible sources of the PS term are still to be discovered. There, the approach suggested in Ref. [22] for the case of the Abelian Higgs model looks especially promising, provided that one manages to identify in the Yang-Mills integration measure those degrees of freedom which can be unambiguously related to the string world-sheet coordinates. 
Funding: This research received no external funding.

Conflicts of Interest: The author declares no conflict of interest.

\section{References}

1. Makeenko, Y.M. Methods of Contemporary Gauge Theory; Cambridge University Press: Cambridge, UK, 2002.

2. Antonov, D. Monopole-based scenarios of confinement and deconfinement in 3D and 4D. Universe 2017, 3, 50. [CrossRef]

3. Wilson, K.G. Confinement of quarks. Phys. Rev. D 1974, 10, 2445-2459. [CrossRef]

4. Dosch, H.G. Gluon condensate and effective linear potential. Phys. Lett. B 1987, 190, 177-181. [CrossRef]

5. Marquard, U.; Dosch, H.G. Potential and sum-rule approach in QCD. Phys. Rev. D 1987, 35, $2238-2243$. [CrossRef] [PubMed]

6. Dosch, H.G.; Simonov, Y.A. The area law of the Wilson loop and vacuum field correlators. Phys. Lett. B 1988, 205, 339-344. [CrossRef]

7. Polchinski, J.; Strominger, A. Effective string theory. Phys. Rev. Lett. 1991, 67, 1681-1684. [CrossRef] [PubMed]

8. Polyakov, A.M. Gauge Fields and Strings; Harwood Academic Publishers: Chur, Switzerland, 1987.

9. Lüscher, M. Symmetry-breaking aspects of the roughening transition in gauge theories. Nucl. Phys. B 1981, 180, 317-329. [CrossRef]

10. Alvarez, O. Static potential in string models. Phys. Rev. D 1981, 24, 440-449. [CrossRef]

11. Antonov, D. String nature of confinement in (non-)Abelian gauge theories. Surv. High Energy Phys. 2000, 14, 265-355. [CrossRef]

12. Antonov, D. Nonperturbative Methods in Gauge Theories; Pisa University Press: Pisa, Italy, 2013.

13. Kornelis, W.; Dosch, H.G. Higher cumulants in the cluster expansion in QCD. Nucl. Phys. Proc. Suppl. 2001, 96, 426-431. [CrossRef]

14. Meggiolaro, E. Field strength correlators in QCD: New fits to the lattice data. Phys. Lett. B 1999, 451, 414-421. [CrossRef]

15. Di Giacomo, A.; Dosch, H.G.; Shevchenko, V.I.; Simonov, Y.A. Field correlators in QCD: Theory and applications. Phys. Rept. 2002, 372, 319-368. [CrossRef]

16. Antonov, D.; Ebert, D. Confining properties of Abelian(-projected) theories. Eur. Phys. J. C 2000, 12, 349-359. [CrossRef]

17. Karabali, D.; Kim, C.; Nair, V.P. On the vacuum wavefunction and string tension of Yang-Mills theories in (2+1) dimensions. Phys. Lett. B 1998, 434, 103-109. [CrossRef]

18. Nair, V.P. Three ideas on magnetic mass. arXiv 1998, arXiv:hep-th/9809086.

19. Shifman, M.A.; Vainshtein, A.I.; Zakharov, V.I. QCD and resonance physics. Theoretical foundations. Nucl. Phys. B 1979, 147, 385-447. [CrossRef]

20. Bialas, P.; Daniel, L.; Morel, A.; Petersson, B. Thermodynamics of SU(3) gauge theory in $2+1$ dimensions. Nucl. Phys. B 2009, 807, 547-565. [CrossRef]

21. Bialas, P.; Daniel, L.; Morel, A.; Petersson, B. Three-dimensional finite temperature SU(3) gauge theory in the confined region and the string picture. Nucl. Phys. B 2010, 836, 91-99. [CrossRef]

22. Akhmedov, E.T.; Chernodub, M.N.; Polikarpov, M.I.; Zubkov, M.A. Quantum theory of strings in an Abelian Higgs model. Phys. Rev. D 1996, 53, 2087-2095. [CrossRef] [PubMed]

(C) 2020 by the author. Licensee MDPI, Basel, Switzerland. This article is an open access article distributed under the terms and conditions of the Creative Commons Attribution (CC BY) license (http://creativecommons.org/licenses/by/4.0/). 